\title{
Pump phase transfer and its control in hybrid seeded optical parametric amplifiers
}

\author{
Peng Yuan ${ }^{1}$, Jingui $\mathrm{Ma}^{1}$, Yongzhi Wang ${ }^{2}$, Jing Wang ${ }^{1}$, Daolong Tang ${ }^{1}$, and Liejia Qian ${ }^{1}$ \\ ${ }^{1}$ Key Laboratory for Laser Plasma (Ministry of Education) and Department of Physics and Astronomy, Shanghai Jiao Tong University, \\ Shanghai 200240, China \\ ${ }^{2}$ Department of Optical Science and Engineering, Fudan University, Shanghai 200433, China \\ (Received 16 June 2014; revised 30 July 2014; accepted 19 August 2014)
}

\begin{abstract}
Due to the existence of spatial walk-off and/or group-velocity mismatch effects, pump-to-signal phase transfer becomes inevitable during parametric amplification. We experimentally demonstrate that in hybrid seeded optical parametric amplifiers (OPAs) that include two OPA stages seeded by the signal and idler waves, respectively, the phase of the output signal can be restored to its initial value, although there are spatial and temporal phase fluctuations on the pump source. This method significantly relaxes the requirement for high pump beam quality, which is always very stringent in parametric amplification systems. With the introduction of this scheme into birefringent phase-matching OPAs or chirped-pulse OPAs, it should be promising to achieve intense femtosecond laser pulses that are close to the diffraction limit in space and ultra-high contrast in time, simultaneously.
\end{abstract}

Keywords: hybrid seeding; parametric amplifier; pump-to-signal phase transfer

\section{Introduction}

Optical parametric amplification (OPA) has demonstrated unique flexibility in producing femtosecond laser pulses tunable from 0.2 to $20 \mu \mathrm{m}^{[1]}$. To increase the availability of ultra-short pulses with higher peak power, the scheme of chirped-pulse amplification (CPA) was introduced ${ }^{[2]}$. The integration of OPA and CPA into optical parametric chirpedpulse amplification (OPCPA) has rapidly pushed the peak power of amplified ultra-short laser pulses up to the petawatt level ${ }^{[3]}$. The development has benefited from some important advantages of OPCPA with respect to conventional CPA systems based on laser gain media: (i) the parametric gain within a single pass through a nonlinear crystal can be as high as many tens of decibels, minimizing the Bintegral and allowing simple and compact setups ${ }^{[4]}$; (ii) parametric amplification supports much wider gain bandwidth $(>100 \mathrm{~nm})$, enabling the production of pulse durations in the few-cycle regime; (iii) there are few thermal loading effects during parametric amplification due to the weak parasitic absorption, ensuring good beam quality of the amplified signal. However, parametric amplification has a disadvantage that should not be ignored - its requirement for pump beam quality is much more stringent than that

Correspondence to: Jing Wang, Key Laboratory for Laser Plasma and Department of Physics and Astronomy, Shanghai Jiao Tong University, Shanghai 200240, China. Email: wangj1118@sjtu.edu.cn for conventional $\mathrm{CPA}^{[3]}$. During parametric amplification, any temporal modulations and random noise on the pump beam would be imparted to the signal and idler pulses due to the intensity-independent parametric gain. The contrast degradation of the amplified signal due to pump noise transfer has been theoretically and experimentally verified ${ }^{[5,6]}$. However, the fact of pump-to-signal phase transfer, which could degrade the beam quality and temporal contrast of the amplified output severely, has been less appreciated.

During the parametric amplification process without an initial idler beam, the phases of the pump (at frequency $\omega_{p}$ ), signal $\left(\omega_{s}\right)$ and idler $\left(\omega_{i}\right)$ waves obey ${ }^{[7]}$

$$
\begin{aligned}
\phi_{s} & =\phi_{s}(0), \\
\phi_{i} & =\pi / 2+\phi_{p}-\phi_{s},
\end{aligned}
$$

under the conditions of phase matching and no spatial walkoff or group-velocity mismatch (GVM) effects. At this point, the phase of the amplified signal, $\phi_{s}$, is independent of the pump phase, $\phi_{p}$, while the phase of the newly generated idler wave, $\phi_{i}$, accommodates the phase difference between the pump and signal. This phase relation has been widely adopted as a nonlinear approach to control the carrierenvelope phase of ultra-fast light pulses ${ }^{[8]}$. However, when there is spatial walk-off and/or a temporal GVM effect, the phase relations are no longer valid, and both the signal and idler waves will partake of the pump phase in a very 
(a)

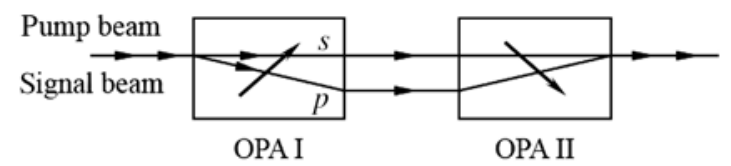

(b)

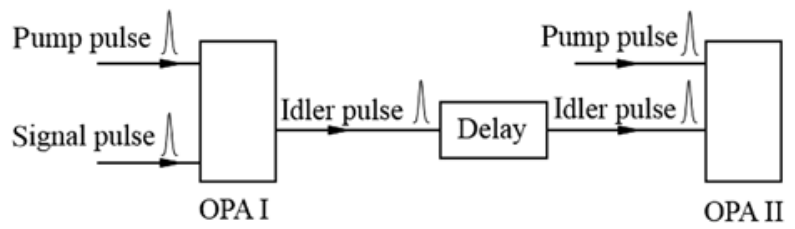

Figure 1. Schematic of optimized OPA designs: (a) walk-off-compensating configuration; (b) hybrid seeding configuration (the two-stage OPAs are seeded by signal and idler waves, respectively).

sophisticated manner. That is, the phase distortion will transfer from the pump laser to the amplified signal.

For parametric amplification based on quasi-phase matching (QPM) using a type- $0\left(e_{p} \rightarrow e_{s}+e_{i}\right)$ scheme, there is no spatial walk-off between interacting waves and, hence, no spatial phase transfer. A QPM parametric amplifier could thus use a multi-mode laser as the pump source ${ }^{[9,10]}$. For parametric amplifiers adopting birefringent nonlinear crystal in which phase matching relies on the presence of both ordinary and extraordinary waves, spatial walk-off always exists and so does spatial phase transfer. This problem could be counteracted by using pairs of crystals arranged in a walkoff-compensated orientation ${ }^{[11]}$, as shown in Figure 1(a), where the walk-off direction is reversed in the second crystal of each pair. However, there is no analogous method in the temporal domain that can eliminate the influence of GVM. Once the wavelengths of the interacting waves and the nonlinear crystal are chosen, the GVM relations (including its sign and value) are determined as well. Thus, reversal of the sign of GVM in a second OPA stage is much more difficult than reversal of the walk-off direction.

In this paper, we explore an approach toward controlling the pump-to-signal phase transfer in both the spatial and temporal domains. This scheme, as illustrated by Figure 1(b), is also known as hybrid seeded OPAs. It includes two OPA stages which are seeded by the signal and idler waves respectively. Hybrid seeding was previously adopted to eliminate the background of initial $\mathrm{CW}$ injection ${ }^{[12]}$ or the undesired preceding and trailing oscillator pulses in the injection ${ }^{[13]}$. Here, we experimentally demonstrate that hybrid seeding can make it possible to restore the phase of the output signal to its initial value, regardless of the existence of GVM and walk-off effects in each OPA stage and the presence of both spatial and temporal phase distortion on the pump laser. Both the spatial beam quality and the temporal contrast of the amplified signal can thus be significantly improved as the pump phase transfer is blocked. Due to the tolerance of both spatial and temporal pump phase aberrations that normally accompany high energy pump lasers, the proposed method

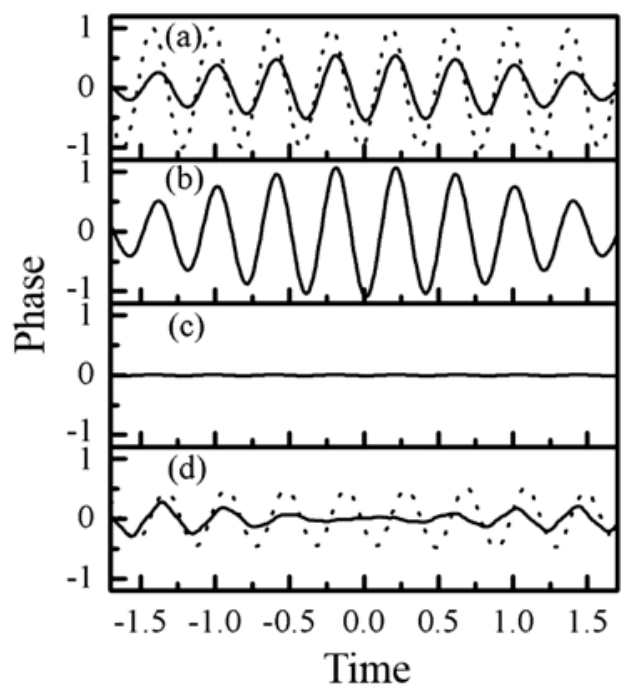

Figure 2. Calculated signal phase distortion and restoration in a two-stage OPA system. (a) The dotted line plots the initial phase modulation on the pump pulse. The solid line is the phase of the amplified signal from the first stage OPA. (b) The phase of the amplified signal from the second OPA stage which is seeded by the amplified signal from the first stage (conventional multistage OPA configuration). (c) The phase of the amplified signal from a hybrid seeded OPA with the condition $L_{\mathrm{GVM}(i-p)}=L_{\mathrm{GVM}(s-p)}=L_{1}=$ $L_{2}$. (d) The phase of the amplified signal (solid line) from a hybrid seeded OPA with the condition $L_{\mathrm{GVM}(i-p)}=1.5 L_{\mathrm{GVM}(s-p)}=3 L_{1}=2 L_{2}$. The dotted line in (d) is the phase of the pump pulse after amplification. The nonlinear length $L_{\mathrm{NL}}{ }^{[14]}$, as an identification of the pump intensity, was fixed in the calculations to be $L_{\mathrm{NL}}=0.3 L_{1}$ for each stage.

may provide a feasible route for the construction of high quality intense laser sources based on OPCPA.

\section{Numerical simulations}

To start with, we simulate the behavior of pump-to-signal phase transfer in a two-stage OPA using the standard coupled-wave equations ${ }^{[14]}$. Identical sinusoidal phase modulation, plotted as the dotted line in Figure 2(a), was introduced into the Gaussian pump pulse. The initial signal pulse having a uniform phase was also assumed to be Gaussian in shape with a duration of $\tau_{s}$. Due to the pulse slippage caused by GVM, the pump phase modulation pattern was imprinted onto the amplified signal, as plotted by the solid line in Figure 2(a). When this distorted signal was injected into a second OPA stage, the phase fluctuation was further enhanced, as indicated by Figure 2(b). It is important to point out that the temporal phase transfer is governed only by the GVM between the pump and the idler (i.e., $\mathrm{GVM}_{i-p}$ ). Only when $\mathrm{GVM}_{i-p} \neq 0$ will the pump phase transfer to the signal. However, the idler wave always inherits the pump phase pattern.

If we seeded the second OPA stage with the pumpphase-imposed idler output from the first stage, complete restoration of the uniform signal phase could be achieved, as shown in Figure 2(c). The restoration conditions used in the calculations included $\mathrm{GVM}_{i-p}=\mathrm{GVM}_{s-p}$ (the GVM 
between pump and idler equals the GVM between pump and signal), $L_{1}=L_{2}$ (the two crystals have equal lengths) and the pump pulse overlapped with the idler well at the entrance of the second OPA stage. In a more general case, the establishment condition of phase restoration can extend to $L_{1} \times \mathrm{GVM}_{i-p}=L_{2} \times \mathrm{GVM}_{s-p}$, as illustrated by Figure 2(d). Even if this condition cannot be exactly satisfied in real systems, the signal phase can still be partially restored and the restoration degree relies on the amount of $\mathrm{GVM}_{s-i}(\mathrm{GVM}$ between the signal and idler). That is, satisfactory control of pump-to-signal phase transfer necessitates a relatively small $\mathrm{GVM}_{s-i}$. An analogous result was also found in the spatial domain. In type-I phase matching $\left(e_{p} \rightarrow o_{s}+o_{i}\right)$ OPAs, both the signal and the idler are ordinary waves. Hence, there would not be spatial walk-off between the signal and the idler. Complete restoration of the spatial phase of the signal beam is thus easy to achieve.

\section{Experimental setup and results}

To experimentally demonstrate the capability of controlling phase transfer by hybrid seeding, a two-stage femtosecond OPA system was employed, as shown in Figure 1(b). Two $10 \mathrm{~mm}$-thick uncoated $\mathrm{MgO}: \mathrm{LiNbO}_{3}$ crystals were used in type-I phase matching. A commercial femtosecond Ti:sapphire laser oscillator-regenerative amplifier (SpectraPhysics, Spitfire) producing $500 \mu \mathrm{J}, 70$ fs laser pulses at $800 \mathrm{~nm}$ with a repetition rate of $1 \mathrm{kHz}$ was adopted as the pump source. A $0.5 \mathrm{~W} 1064 \mathrm{~nm}$ diode-pumped CW Nd: $\mathrm{YVO}_{4}$ laser operating in $\mathrm{TEM}_{00}$ mode served as the seed source. The pump beam was divided into two by a beam splitter with a reflectance of $\sim 40 \%$. These two beams were then telescoped and collimated to obtain equal intensities of $\sim 90 \mathrm{GW} \mathrm{cm}{ }^{-2}$ for pumping of the two OPA stages. The beam profile of the collimated pump is shown in Figure 3(a). In order to optimize the OPA gain and conversion efficiency, a negative chirp was deliberately introduced to the pump pulse to obtain a duration of $\sim 150 \mathrm{fs}$ for dispersion compensation ${ }^{[15]}$. This pulse duration as well as the $70 \mathrm{fs}$ mentioned before was measured using a homemade self-correlator. The OPA was tuned to operate at a signal wavelength of $\sim 1040 \mathrm{~nm}$, where the signal and idler have approximately the same value of group velocity. Thus, control of the phase transfer in both the temporal and spatial domains is possible by using this hybrid seeding scheme.

In the spatial domain, the beam divergence caused by the Kerr effect was regarded as the spatial phase distortion of the pump laser in our experiment. Due to the presence of a significant accumulated Kerr nonlinear phase shift $\left(\Delta \varphi_{n l} \sim 6 \pi\right)$ in each OPA crystal, strong whole-beam self-focusing occurred across the pump beam, as conveyed by Figure 3(b). A small-size self-focusing effect was not observed, which is reasonable as the beam profile was very smooth. Since pump-to-signal phase transfer would occur only within the walk-off plane, the signal beam from the first OPA stage was found to be highly astigmatic, as shown in
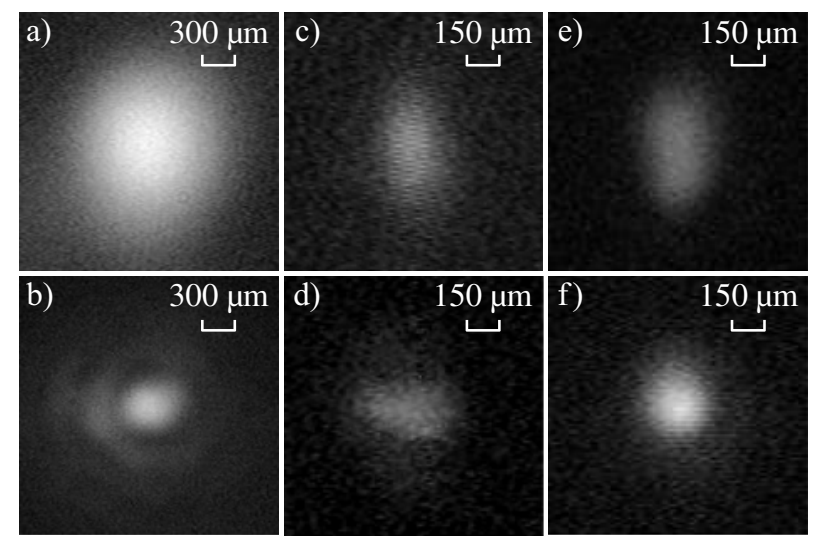

Figure 3. Beam patterns of the pump beam measured $\sim 400 \mathrm{~mm}$ after the crystal (used for Kerr-effect-induced spatial phase distortion): (a) in the linear propagation regime; (b) at high intensity $\left(90 \mathrm{GW} \mathrm{cm}^{-2}\right)$. Beam patterns of the amplified signal from the first OPA stage measured at the focus: (c) in the walk-off plane; (d) in the no-walk-off plane. Amplified signal from the second OPA stage measured at the focus in the no-walkoff plane: when the overlap between the pump and idler beams was not optimized (e) and when it was optimized (f).

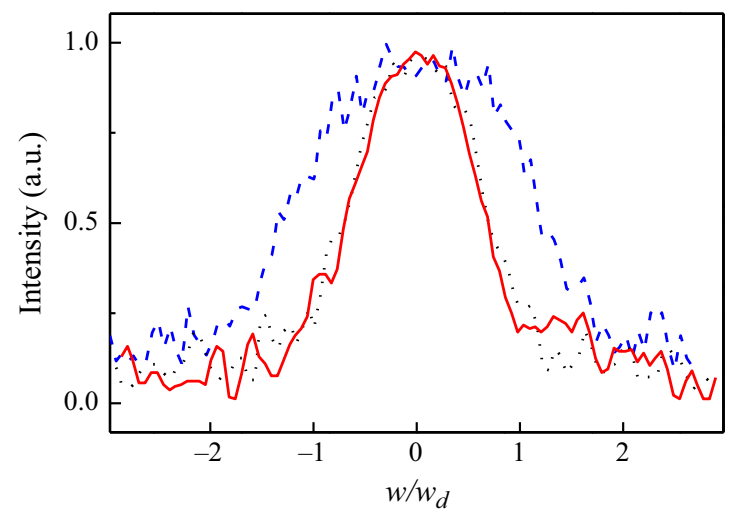

Figure 4. Measured beam profiles of the amplified signal from the hybrid seeded OPA. The dotted line plots the beam profile in the no-walk-off plane. The solid line is the beam profile in the walk-off plane after the spatial overlap between the pump and idler beams was optimized. The dashed line shows the beam profile in the walk-off plane when the pump-idler overlap was not optimized.

Figures 3(c) and (d). This astigmatism would become more severe if the signal were amplified further in a second OPA stage. By seeding the second stage with the idler from the first stage instead of the signal, although the output signal beam is also astigmatic in general (Figure 3(e)), a quite satisfactory compensation of the astigmatism (Figure 3(f)) can be obtained by adjusting the seeding idler beam to make it coincide with the pump beam in space at the entrance of the second OPA stage. As plotted in Figure 4, the astigmatism induced by pump phase transfer in the walkoff plane (solid line) was compensated close to the level of that in the anastigmatic no-walk-off dimension (dotted line). This indicates that the output signal beam of the hybrid seeded OPA could be very close to its diffraction limit. It should be pointed out that hybrid seeding would not reduce 

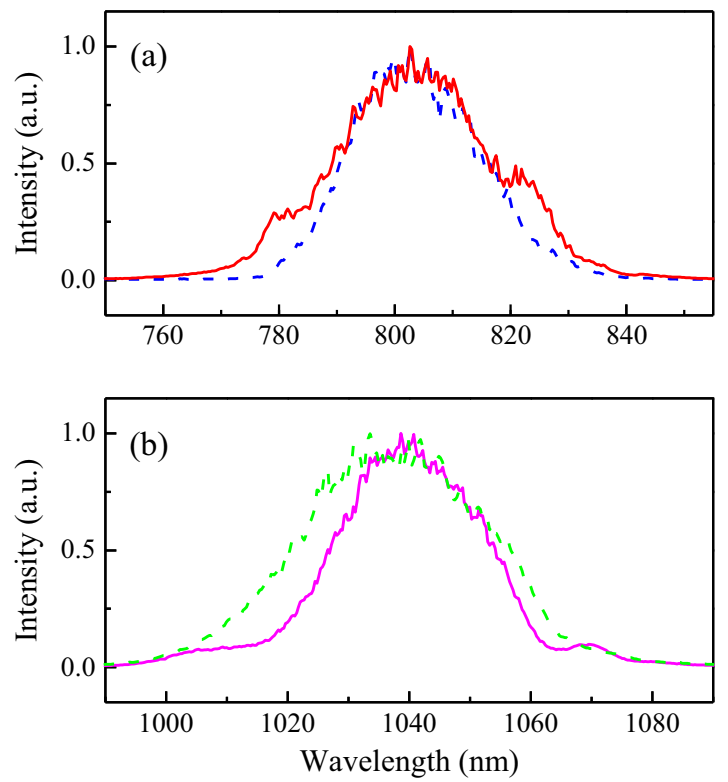

Figure 5. (a) Measured pump pulse spectra before (dashed line) and after (solid line) the crystal (where self-phase modulation occurred). (b) Measured signal pulse spectra when the time delay between the idler and the pump was $\sim 150$ fs (dashed line) and 0 (solid line), respectively.

the conversion efficiency. The obtained signal pulse energy of $\sim 20 \mu \mathrm{J}$ was similar to that obtained by seeding both stages with the signal wave. The pump-to-signal conversion efficiency was $\sim 5 \%$. These experimental results clearly verify the capability of the hybrid seeded OPA scheme in signal phase restoration in the spatial domain.

To introduce some temporal phase distortion into the pump, the self-phase modulation effect when a high intensity pump laser passes through a nonlinear crystal was used. Figure 5(a) shows the pump spectrum before and after the crystal. Spectral broadening was observed, which indicated the occurrence of the desired self-phase modulation. During parametric amplification, the pump phase modulation was imparted to the signal pulse and led to signal spectrum broadening, as indicated by the dashed line in Figure 5(b). By seeding the second OPA stage with the idler pulse from the first stage and synchronizing the idler with the pump pulse carefully, a phase-restored signal pulse could be obtained, as indicated by the relatively narrower spectrum (solid line) in Figure 5(b).

\section{Conclusion}

We have demonstrated that by using a hybrid seeded OPA scheme, the phase distortion induced by pump phase transfer during parametric amplification can be well controlled in both the spatial and the temporal domain. To achieve higher output power, the two-stage configuration employed in our work could be further extended to several pairs of crystals, where the second crystal of each pair is seeded with the idler wave from the first crystal. In this way, the phase transfer in the second crystal could exactly cancel the phase transfer in the first crystal. That is, the pump-to-signal phase transfer can no longer accumulate with increase of the nonlinear interaction length. Moreover, the hybrid seeded OPA has almost the same conversion efficiency relative to classical multistage OPAs of the same overall length. Up to now, OPCPA systems have always resorted to various nonlinear approaches for pulse contrast enhancement, which have very low energy efficiency $\sim 10 \%$. By using the proposed method in OPCPA systems, the output temporal contrast can be enhanced while not sacrificing the amplified signal energy at all. We believe that this work provides new insight into the optimized design of further femtosecond OPA or OPCPA systems.

\section{References}

1. M. H. Dunn and M. Ebrahimzadeh, Science 286, 1513 (1999).

2. A. Dubietis, G. Jonusauskas, and A. Piskarskas, Opt. Commun. 88, 437 (1992).

3. E. A. Khazanov and A. M. Sergeev, Phys. Usp. 51, 969 (2008).

4. Z. Xu, X. Yang, Y. Leng, H. Lu, L. Lin, Z. Zhang, R. Li, W. Zhang, D. Yin, S. Jin, J. Peng, B. Tang, and B. Zhao, Chin. Opt. Lett. 01, 24 (2003).

5. N. Forget, A. Cotel, E. Brambrink, P. Audebert, C. L. Blanc, A. Jullien, O. Albert, and G. Chériaux, Opt. Lett. 30, 2921 (2005).

6. I. N. Ross, G. H. C. New, and P. K. Bates, Opt. Commun. 273, 510 (2007).

7. I. N. Ross, P. Matousek, G. H. C. New, and K. Osvay, J. Opt. Soc. Am. B 19, 2945 (2002).

8. A. Baltuska, K. Fuji, and T. Kobayashi, Phys. Rev. Lett. 88, 133901 (2002).

9. A. Galvanauskas, A. Hariharan, D. Harter, M. A. Arbore, and M. M. Fejer, Opt. Lett. 23, 210 (1998).

10. I. Jovanovic, J. R. Schmidt, and C. A. Ebbers, Appl. Phys. Lett. 83, 4125 (2003).

11. D. J. Armstrong, W. J. Alford, T. D. Raymond, A. V. Smith, and M. S. Bowers, J. Opt. Soc. Am. B 14, 460 (1997).

12. H. Luo, L. Qian, P. Yuan, H. Zhu, and S. Wen, Opt. Express 13, 9747 (2005).

13. I. Jovanovic, C. P. J. Barty, C. Haefner, and B. Wattellier, Opt. Lett. 31, 787 (2006).

14. P. Zhu, L. Qian, S. Xue, and Z. Lin, Opt. Laser Technol. 35, 13 (2003).

15. P. Yuan, L. Qian, H. Luo, H. Zhu, and S. Wen, IEEE J. Sel. Top. Quantum Electron. 12, 181 (2006). 